\title{
Vascular Thrombosis
}

National Cancer Institute

\section{Source}

National Cancer Institute. Vascular Thrombosis. NCI Thesaurus. Code C114397.

The formation of a blood clot in the lumen of a vessel; causes include coagulation disorders, and vascular endothelial injury. 